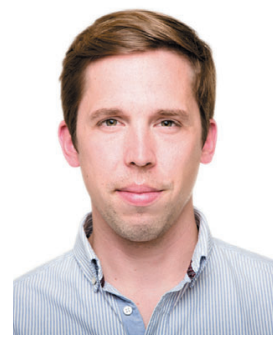

Matthias Raspe

Koordination «PneumoCampus»

\section{Von Zukunftsgestaltung und digitalen Tools}

\begin{abstract}
Mit einer Patientin, die wegen einer langsam zunehmenden Dyspnoe und rechtsseitigem Pleuraerguss zur weiteren Abklärung stationär aufgenommen wurde, befassen sich die Blickdiagnosen dieser Ausgabe. Die Auflösung dieses spannenden Falls finden Sie wieder auf der KARGER-KOMPASS-PNEUMOLOGIE-Webseite.

Die Arbeitsgemeinschaft junge DEGRO (jDEGRO) ist Interessensvertretung der Nachwuchswissenschaftlerinnen und -wissenschaftler in der Radioonkologie und gestaltet Forschung und Weiterbildung innerhalb der Fachgesellschaft aktiv mit. In ihrem Beitrag stellen Mitglieder der jDEGRO u.a. die Arbeitsgemeinschaft vor und schildern die erfolgreiche Bildung eines deutschlandweiten Forschungsnetzwerks, das in einem ersten Projekt Aspekte der Bestrahlung des inoperablen nicht kleinzelligen Lungenkarzinoms (NSCLC) im Stadium III untersuchte.

Außerdem berichtet uns Dr. Tobias Müller, Leiter der Stabsstelle Digitale Transformation bei der Rhön-Klinikum AG und wissenschaftlicher Mitarbeiter am Zentrum für unerkannte und seltene Erkrankungen am Universitätsklinikum Marburg, über digitale Tools zur Unterstützung der Differenzialdiagnose, die für Ärzte und Patienten zur Verfügung stehen. Und zu guter Letzt finden Sie einen weiteren «Überlebenstipp für die Weiterbildung».
\end{abstract}

\section{Blickdiagnosen \\ Welche Diagnose vermuten Sie?}

Die 42-jährige Patientin fiel mit einer langsam zunehmenden Dyspnoe und rechtsseitigem Pleuraerguss auf und wurde zur weiteren Abklärung stationär eingewiesen. Anamnestisch bestanden keine relevanten Vorerkrankungen und es wurden keine Medikamente regelmäßig eingenommen. Die Patientin war Nichtraucherin mit etwa 12 Packungsjahren und arbeitete im Außenhandel. In der körperlichen Untersuchung fielen eine zentrale Zyanose und Zeichen der Herzinsuffizienz (Aszites, Beinödeme) auf. Laborchemisch imponierten ein deutlich erhöhtes NTproBNP und erhöhte Leberenzyme. Im Rahmen der Abklärung wurden unter anderem die beiden folgenden Bildbefunde erhoben ( $\mathrm{A}$ und $\mathrm{B}$ ).

Auflösung unter:

www.karger.com/blickdiagnose-kkp-2-2019

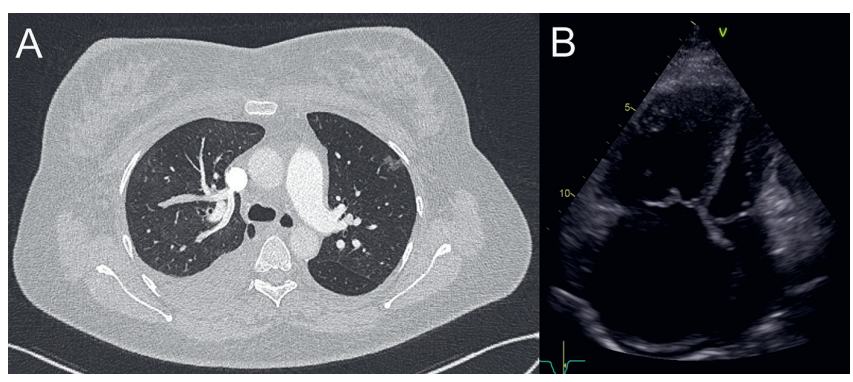

Bildbefunde mit freundlicher Genehmigung von PD Dr. med. C. Althoff, Leitung Interventionsradiologie Charité Campus Mitte, Institut und Klinik für Radiologie CCM und CVK, Charité - Universitätsmedizin Berlin.

Kontaktadresse: Dr. Matthias Raspe, Medizinische Klinik mit Schwerpunkt Infektiologie und Pneumologie, Charité - Universitätsmedizin Berlin, Augustenburger Platz 1, 13353 Berlin, Deutschland, matthias. raspe@charite.de

\title{
KARGER
}

() 2019 S. Karger GmbH, Freiburg 
In den letzten Jahrzehnten hat sich die medizinische (Arbeits-)Welt und mit ihr die Einstellungen und Erwartungen junger Ärzte signifikant verändert [1, 2]. Umso wichtiger ist es, die Anliegen der jungen Generation konzertiert und effektiv zu artikulieren.

Innerhalb der Deutschen Gesellschaft für Radioonkologie (DEGRO) nimmt die AG junge DEGRO (jDEGRO) diese Aufgabe erfolgreich wahr. Als Zusammenschluss junger Strahlentherapeut/inn/en, Medizinphysiker/innen und Strahlenbiolog/inn/en versteht sie sich als offenes Netzwerk, das den forschungsinteressierten Nachwuchs aus den eigenen Reihen fördern sowie Weiterbildung und Forschung optimieren will.

Im Hinblick auf klinische Forschungsprojekte gelang der AG die erfolgreiche Bildung eines deutschlandweiten Forschungsnetzwerks, das in einem ersten Projekt die Bestrahlung des inoperablen nicht kleinzelligen Lungenkarzinoms (NSCLC) im Stadium III untersuchte: Hierbei interessierte die Studiengruppe die prognostische Aussagekraft des Tumorvolumens (gross tumor volume, GTV), das bei der Radiotherapieplanung auf Grundlage der CT- bzw. PET-CTBildgebung definiert wird. Vorab wurden in einer systematischen Literaturrecherche Vorarbeiten identifiziert und analysiert [3].

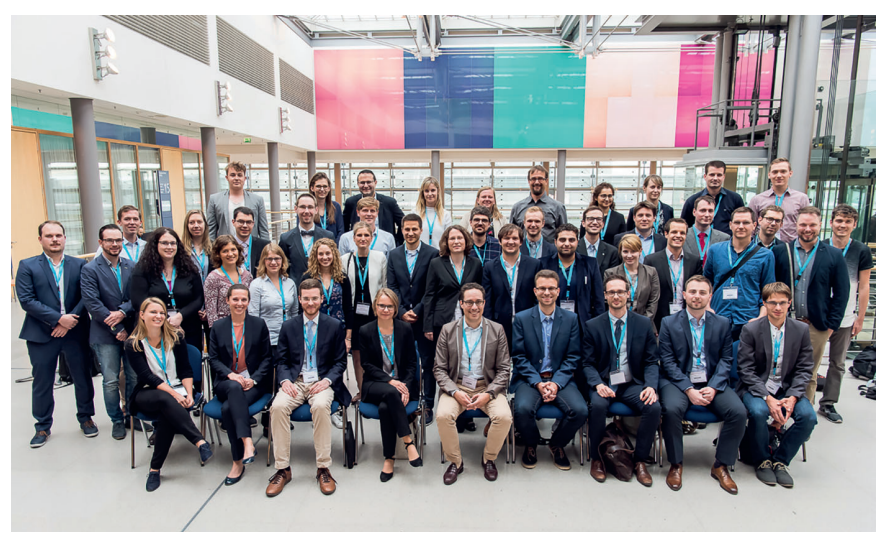

Gruppenfoto der jDEGRO auf der Jahrestagung der Deutschen Gesellschaft für Radioonkologie (Leipzig 2018).

An der folgenden multizentrischen Studie (NCT03055715; ARO2017-01) beteiligten sich 21 Zentren, eingeschlossen waren darin 346 Patienten [4]. Mittels standardisierter elektronischer «case report forms» wurden das initiale GTV sowie seine Änderung im Verlauf erfasst. Dies erfolgte anhand einer weiteren Schnittbildgebung vor Beginn der Boost-Bestrahlung (nach etwa 5 Wochen Therapie), anhand derer ein zweites GTV eingezeichnet wurde.

Neben dem GTV zu Beginn der Bestrahlung hatte v.a. das im Verlauf der Radiotherapie neu erstellte GTV einen signifikanten Einfluss auf das Gesamtüberleben, unabhängig von anderen Prognosefaktoren wie dem T- oder N-Stadium. Auch die mittels Mediansplit eingeteilte absolute Volumenreduktion vom ersten zum zweiten GTV hatte einen signifikanten Einfluss auf das Gesamtüberleben [5]. Dies zeigt die prognostische Wertigkeit des (intratherapeutischen)

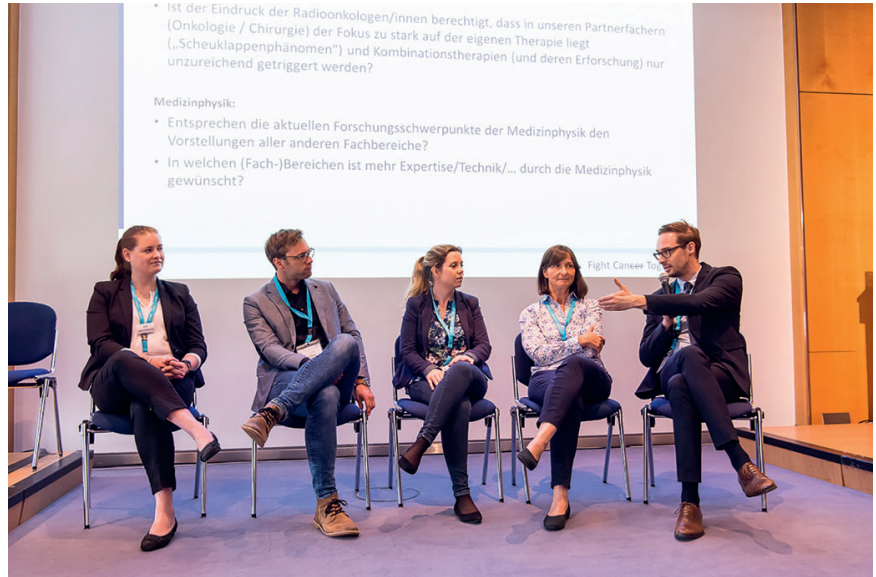

Im Symposium der AG jDEGRO «Young Professionals Fight Cancer - Perspektiven von Nachwuchsgruppen in der Onkologie» auf dem Jahreskongress 2018.

GTV bei inoperablen NSCLC-Patienten. Ausgehend hiervon sind aufbauende Analysen unter Einschluss der Bilddaten und Bestrahlungspläne geplant.

Neben der (klinischen) Forschung gehört es zu den AG-Aufgaben, die Jahrestagungen der DEGRO mitzugestalten: Mitglieder der jDEGRO sind stets Teil des Organisationsteams und vermitteln die Beteiligung am wissenschaftlichen Programm. Zusammen mit den Kongresspräsidenten werden die Themen ausgewählt, das Programm konzipiert und organisatorische Details diskutiert. Zusätzlich werden durch Mitglieder der jDEGRO Tagungsbeiträge begutachtet, wissenschaftliche Sitzungen moderiert und eigene Veranstaltungen organisiert. Die jDEGRO deckt damit vielfältige Aufgaben ab, die wichtig sind, um den Jahreskongress für «alte Hasen» und «Neulinge» der Strahlentherapie in gleichem Maße interessant zu gestalten. Weiterhin beteiligt sich die jDEGRO daran, die Facharztausbildung und das Fortbildungsangebot stetig weiterzuentwickeln. Eine deutschlandweite Umfrage unserer AG zur Facharztausbildung in

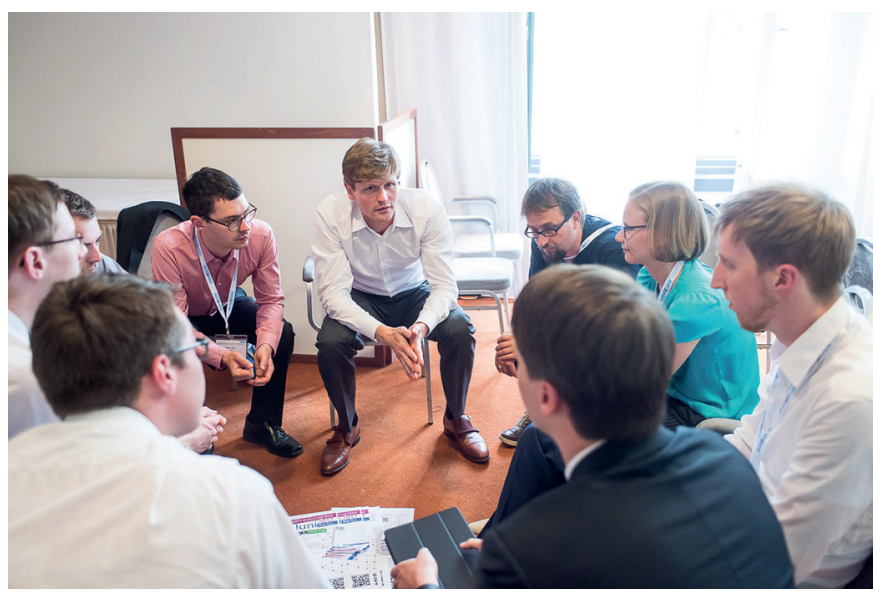

Teamwork im Rahmen der AG-Sitzung der jDEGRO. 
der Strahlentherapie zeigte, dass junge Kolleg/inn/en insgesamt zufrieden in ihrem Fach sind und die Qualität der Weiterbildung schätzen [6]. Insbesondere bei Spezialtechniken und seltenen Indikationen wünschen sich jedoch viele Kolleg/inn/en weitere Angebote, sodass ein deutschlandweites Hospitationsprogramm zum Zwecke der fokussierten Weiterbildung in diesen Bereichen in Planung ist. Zusätzlich läuft aktuell eine Umfrage zum Stand der strahlentherapeutischen Lehre an den deutschen Universitätskliniken, auch in Hinblick auf die Entwicklung des Nationalen Lernzielkatalogs Medizin und die Umsetzung des Masterplans Medizinstudium 2020.

Diese vielfältigen Aufgaben werden in den 3 jährlichen AG-Sitzungen der jDEGRO und regelmäßigen Telefonkonferenzen besprochen und koordiniert. In den angeregten Diskussionen werden konstruktive Lösungen im kollegialen Austausch erarbeitet. Das bisher Erreichte ist für die Gruppe Ansporn, an der Zukunft des Faches Strahlentherapie weiterhin engagiert gestaltend mitzuarbeiten.

\section{Zusammenfassung}

Der AG jDEGRO gelang mit der multizentrischen Studie zur GröBenänderung des Tumorvolumens von NSCLC-Patienten unter Radiotherapie die Etablierung eines leistungsstarken Forschungsnetzwerkes junger Strahlentherapeut/inn/en, -biolog/inn/en und -physiker/innen. Primäre Aufgabe und Anspruch der AG ist es, Forschung und Weiterbildung in der Radioonkologie innerhalb der Fachgesellschaft aktiv mitzugestalten und die Interessen der Nachwuchswissenschaftler/innen zu vertreten und zu fördern.

\section{Literatur}

1 Boysen PG, Daste L, Northern T: Multigenerational Challenges and the Future of Graduate Medical Education. Ochsner J 2016;16:101-107.

2 Desy JR, Reed DA, Wolanskyj AP: Milestones and Millennials: A Perfect PairingCompetency-Based Medical Education and the Learning Preferences of Generation Y. Mayo Clin Proc 2017;92:243-250.

3 Käsmann L, Niyazi M, Blanck O, et al.: Predictive and prognostic value of tumor volume and its changes during radical radiotherapy of stage III non-small cell lung cancer : A systematic review. Strahlenther Onkol 2018;194(2):79-90.

4 Ostheimer C, Baues C, Baumann R, et al.: Predictive value of GTV in radiotherapy of NSCLC - early results of the NCT03055715 trial. Radiotherapy and Oncology 2018;127, S175, 4OC-0329.

5 Ostheimer C, jDEGRO Trial Group: Prognostischer Wert des Tumorvolumens in der Radiochemotherapie des lokal fortgeschrittenen nichtkleinzelligen Bronchialkarzinoms. Pilotstudie der AG Junge DEGRO der Deutschen Gesellschaft für Radioonkologie (DEGRO). FORUM 2018;33:446-447.

6 Dietzel CT, Jablonska K., Niyazi M, et al.: Quality of training in radiation oncology in Germany: where do we stand?: Results from a 2016/2017 survey performed by the working group «young DEGRO» of the German society of radiation oncology (DEGRO). Strahlenther Onkol 2018;194(4):293-302.

\section{jDEGR๑}

Kontakt:

Dr. Michael Oertel, Klinik für Strahlentherapie - Radioonkologie, Universitätsklinikum Münster, Albert-Schweitzer-Campus 1, Gebäude A1, 48149 Münster, michael.oertel@ukmuenster.de.

Dr. Daniel F. Fleischmann, Klinik und Poliklinik für Strahlentherapie und Radioonkologie, Klinikum der Universität München, LMU München.

Dr. Christian Ostheimer, Klinik und Poliklinik für Strahlentherapie, MartinLuther-Universität Halle-Wittenberg, Halle (Saale).

Dr. Matthias Mäurer, Klinik für Strahlentherapie und Radioonkologie, Universitätsklinikum Jena, Friedrich-Schiller-Universität Jena.

\section{Neue Medien \\ Digitale Tools zur Unterstützung der Differenzialdiagnose für Ärzte und Patienten}

Die Differenzialdiagnose bezeichnet die systematische Betrachtung möglicher Ursachen von Krankheiten und Symptomen [1-3]. Hierbei gilt es, die Befunde aus Anamnese, körperlicher Untersuchung, Labor und apparativer Diagnostik zusammenzuführen und eine Auflistung möglicher Erkrankungen zu erstellen. Mittlerweile gibt es zahlreiche Programme, welche den Arzt bzw. den Patienten bei der Diagnose unterstützen sollen. Mehrheitlich fallen diese unter die Kategorie der «Symptom-Checker», welche auf Basis eingegebener Symptome eine gewichtete Liste mit möglichen Diagnosen ableiten. Obgleich nicht für diesen Zweck entwickelt, ist Google wohl der am meisten verbreitete Symptom-Checker. Egal ob Arzt oder Patient, viele nutzen die Suchmaschine zur Diagnoseunterstützung. Die englischsprachige Google-Version liefert bei Symptomeingabe bereits medizinische Diagnosevorschläge mit jeweiliger relativer Häufigkeitsangabe [4]. Entscheidender Nachteil ist jedoch das intransparente Ranking der Suchergebnisse, die Qualität referenzierter medizinischer Information und mögliche kommerzielle Aspekte. Google wurde als Universalsuchmaschine für das Word Wide Web und nicht für die Aufgabe der Diagnoseunterstützung entwickelt. Für diesen Zweck existieren aber mittlerweile spezielle Anwendungen.
Bereits seit Anfang 2000 am Markt verfügbar ist das englischsprachige und kostenpflichtige Tool «lsabel Healthcare» [5, 6]. Die Symptome können darin im Freitext eingegeben werden. Neben diesen, dem Alter und Geschlecht kann außerdem explizit eine Reiseanamnese eingeben werden. Die Liste mit den möglichen Differenzialdiagnosen ist nach Krankheitsentitäten gegliedert und explizite Notfalldiagnosen werden als «red flag» angezeigt. Ebenfalls im Freitext lassen sich die Symptome bei «Symptoma» eingeben. Basierend auf einer eigenen Ontologie werden Synonyme inkludiert und die Suchbegriffe können weiter spezifiziert werden. Innovativ ist hier die Kombination mit einem Chatbot, welcher nähere Details zur Eingabe erfragt.

Einem anderen Ansatz folgt «visualDx» [7]. Wie der Name vermuten lässt, ist die Benutzerinteraktion bei dieser App visuell geprägt. Das Tool präsentiert dem Benutzer Fotos und Diagramme von Erkrankungen, die sehr hilfreich bei Blickdiagnosen wie z.B. Hauterkrankungen sein können.

Die Schwierigkeiten der Diagnosestellung über digitale Tools werden besonders im Bereich der seltenen Erkrankungen evident. Es ist schlichtweg unmöglich, die häufig variablen Symptomkomplexe der mehr als 8000 seltenen Erkrankungen zu erinnern. In diesem Bereich 
sind 3 Programme explizit zu nennen: «FindZebra» ist eine Suchmaschine speziell für seltene Erkrankungen [8, 9]. Diese indexiert im Gegensatz zu Google nur qualitativ hochwertige und kuratierte Informationen. Die geringe Größe des Index führt zu einer hohen Genauigkeit der Ergebnisse. Eine andere Herangehensweise verfolgt der «Phenomizer» [10, 11]. An der Charité entwickelt, basieren die Diagnosevorschläge hier auf der Auswertung der Human Phenotype Ontology, welche die einzelnen Symptome semantisch verknüpft.

Auch Patientenfotos können in diesem Bereich genutzt werden, wie die App «Face2Gene» zeigt. Diese nutzt Deep-learning-Algorithmen, um Gesichtsmerkmale aus Patientenfotos zu bestimmen und zu quantifizieren, was zu einer gewichteten Liste von Syndromen mit ähnlicher Gesichtsmorphologie führt.

Im Patientenbereich erlangte die App «Ada» durch die Zusammenarbeit mit der Techniker Krankenkasse erhöhte Aufmerksamkeit [12, 13]. Diese App nutzt einen Chatbot, um dialogbasiert die Symptome und Vorerkrankungen des Patienten abzufragen. Beruhend auf dem komplexen Bayes'schen Netz erstellt diese Applikation einen Report für den Anwender, der mögliche Differenzialdiagnosen samt Wahrscheinlichkeiten auflistet, aber auch Triageempfehlungen gibt (z.B. «Notaufnahme aufsuchen», «ärztlichen Rat einholen», «Selbstbehandlung»).

Obwohl die Kombination der Intelligenz von Ärzten mit Anwendungen zur Diagnoseunterstützung ein hohes Potenzial bietet, ist die Evidenzlage noch spärlich. Bezüglich der Vorteile und der diagnostischen Qualität der digitalen Tools existieren bislang nur kleine, rein retrospektive Studien, welche die Genauigkeit aufgrund von Symptomeingaben aus Kasuistiken messen [14-17]. Ebenfalls sind die Diagnosevorschläge stark abhängig von der Nutzereingabe, und kleine Änderungen führen zu abweichenden Ergebnissen. Die Anwendungen existieren primär als eigenständige Software ohne Integration in ärztliche Informationssysteme. Verfügbarkeit und Zugänglichkeit sind auch hier Schlüsselfaktoren. Vor dem Hintergrund fortschreitender Entwicklung kann jedem Kollegen empfohlen werden, sich ebenfalls mit den digitalen Anwendungen zu befassen, denn die Patienten werden diese zukünftig verstärkt nutzen.

\section{Weiterführende Links}

www.isabelhealthcare.com/

www.symptoma.de

www.visualdx.com/

www.findzebra.com/

http://compbio.charite.de/phenomizer/

www.face2gene.com/

https://ada.com/

\section{Literatur}

1 Maude J: Diagnosis (Berl) 2014;1(1):107-109.

2 Bliss M: William Osler. A life in medicine, New York, Oxford University Press, 2007.

3 Jain B: Diagnosis 2017;4:418.

4 Boehret K: www.theverge.com/2016/6/20/11978338/google-symptom-search-appweb-md-health-doctor (letzter Zugriff: 30.01.2019).

5 Nash DB: PT 2010;35(12):651.

6 Henderson EJ, Rubin GP: JRSM Short Rep 2013;4:31.

7 Vardell E, Bou-Crick C: Med Ref S Q 2012;31:414-424.

8 Dragusin R, et al.: Int J Med Inform 2013;82:528-538.

9 Svenstrup D, Jørgensen HL, Winther O: Rare Dis 2015;3:e1083145 (2015).

10 Köhler S, et al.: Am J Hum Genet 2019;85:457-464.

11 Köhler, S. et al.: Nucleic Acids Res 2017;45:D865-D876.

12 Butcher M: https://techcrunch.com/2017/10/31/berlins-ada-health-raises-47m-tobecome-the-alexa-of-healthcare/ (letzter Zugriff: 30.01.2019).

13 www.mobihealthnews.com/content/ada-health-gets- $47 \mathrm{~m}$-ai-powered-chatbottelemedicine-app (letzter Zugriff: 30.01.2019).

14 Bond WF, et al.: J Gen Intern Med 2012;27:213-219.

15 Semigran HL, Linder JA, Gidengil C, et al.: BMJ 2015;351:h3480.

16 Semigran HL, Levine DM, Nundy S et al.: JAMA Intern Med 2016;176:1860-1861.

17 Riches N, et al.: PloS one 2016;11:e0148991.

Dr. Tobias Müller ist Leiter der Stabsstelle Digitale Transformation bei der Rhön-Klinikum AG. Daneben ist der studierte Wirtschaftsinformatiker und Mediziner als wissenschaftlicher Mitarbeiter am Zentrum für unerkannte und seltene Erkrankungen am Universitätsklinikum Marburg tätig.

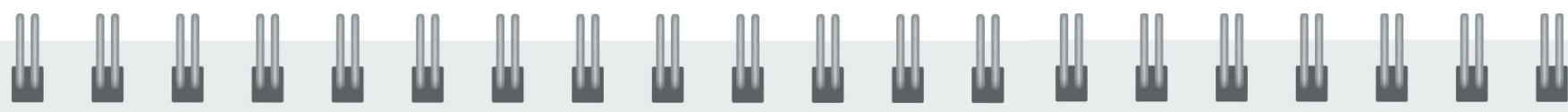

Überlebenstipp für die Weiterbildung

«Eine Weiterbildungsbefugnis muss bei der zuständigen Landesärztekammer beantragt werden. Thre Weiterbildungsbefugte bzw. Ihr Weiterbildungsbefugter hat also für ein bestimmtes Fach und für bestimmte (Zeit-)abschnitte der Weiterbildung eine Berechtigung Sie weiterzubilden. Bevor Sie eine Anstellung antreten, sollten Sie sich über die in der Kliniklabteilung vorhandenen Weiterbildungsberechtigungen informieren. Der einfachste Weg dazu ist über Auskunftsportale der Landesärztekammern im Internet. Insbesondere bei sich ändernden Zuständigkeiten, wie z.B. einem Wechsel in der Klinikleitung, kann es passieren, dass plötzlich die Berechtigungen nicht mehr zu dem passen, was Sie für Thre Weiterbildung brauchen. Hier hilft nur, früh auf das Problem aufmerksam zu werden und sich mit derldem betreffenden Weiterbildungsbefugten abzustimmen.»

Matthias Raspe 